LA-UR-01-2999

Approved for public release; distribution is unlimited.

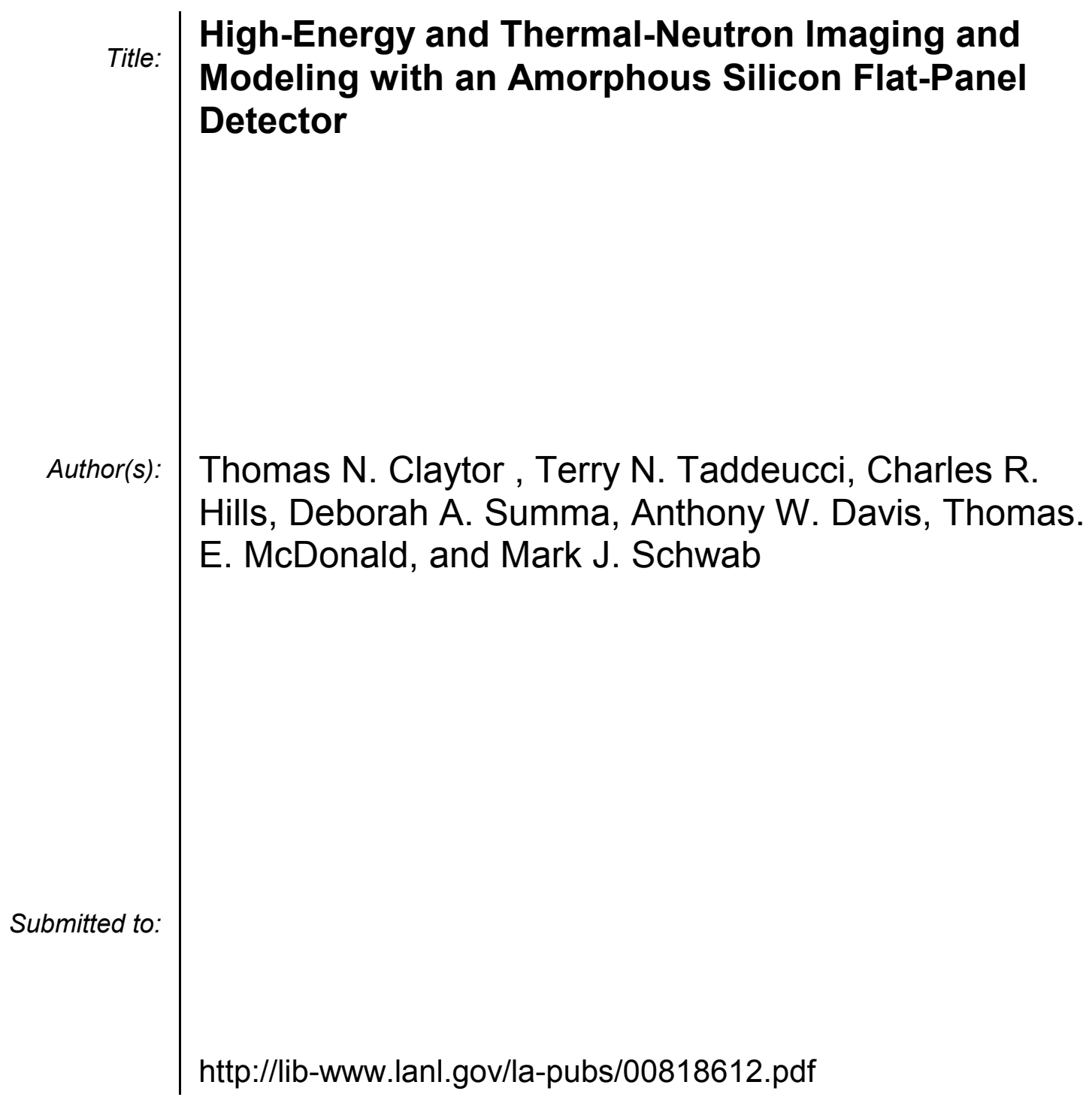

Los Alamos National Laboratory, an affirmative action/equal opportunity employer, is operated by the University of California for the U.S. Department of Energy under contract W-7405-ENG-36. By acceptance of this article, the publisher recognizes that the U.S. Government retains a nonexclusive, royaltyfree license to publish or reproduce the published form of this contribution, or to allow others to do so, for U.S. Government purposes. Los Alamos National Laboratory requests that the publisher identify this article as work performed under the auspices of the U.S. Department of Energy. Los Alamos National Laboratory strongly supports academic freedom and a researcher's right to publish; as an institution, however, the Laboratory does not endorse the viewpoint of a publication or guarantee its technical correctness. 


\title{
High-Energy and Thermal-Neutron Imaging and Modeling with an Amorphous Silicon Flat-Panel Detector
}

\author{
Thomas N. Claytor, Terry N. Taddeucci, Charles R. Hills, Deborah A. Summa, \\ Anthony W. Davis, Thomas. E. McDonald, and Mark J. Schwab \\ Los Alamos National Laboratory \\ Los Alamos NM, 87545
}

\begin{abstract}
The Los Alamos Neutron Science Center (LANSCE) operates two spallation neutron sources dedicated to research in materials science, condensed-matter physics, and fundamental and applied nuclear physics. Prior to 1995, all thermal neutron radiography at Los Alamos was done on a beam port attached to the Omega West reactor, a small $8 \mathrm{MW}$ research reactor used primarily for radioisotope production and prompt and delayed neutron activation analysis. After the closure of this facility, two largely independent radiography development efforts were begun at LANSCE using moderated cold and thermal neutrons from the Target-1 source and high-energy neutrons from the Target- 4 source. Investigations with cold and thermal neutrons employed a neutron converter and film, a scintillation screen and CCD camera system, and a new high-resolution amorphous silicon (a-Si) flat-panel detector system. Recent work with high energy neutrons $\left(E_{n}>1 \mathrm{MeV}\right)$ has involved storage-phosphor image plates. Some comparison high-energy images were obtained with both image plates and the aSi panel and showed equivalent image quality for approximately equal exposure times.
\end{abstract}

Keywords: Tomography, Amorphous Silicon Imager, Storage-Phosphor Image Plates, Neutron Radiography, Radiography Modeling 


\section{Introduction}

Amorphous-silicon (a-Si) flat-panel detector arrays, such as the EG\&G-Heimann RTM 128 [1] or dpiX FS20 or FS33 [2], have proven to be quick and efficient for producing high-quality digital radiographic images for thermal neutron radiography/computed tomography. The pixel size of these a-Si arrays is about $750 \mu \mathrm{m}$ with the RTM and 127 $\mu \mathrm{m}$ with the FS series arrays with a dynamic range in excess of 2800 . In many respects using an a-Si detector is an improvement over other techniques such as CCD cameras, storage-phosphor image plates, or film radiography. Unlike a CCD camera, which is highly susceptible to radiation damage, a-Si detectors can be placed in the beam directly behind the object under examination and do not require any special optics or turning mirrors. The a-Si detector also allows enough data to be acquired to construct a digital image in just a few seconds (minimum gate time $40 \mathrm{~ms}$ ) whereas film or image-plate exposures can take many minutes and need to be digitized with a scanner. The flat panel can, therefore, acquire a complete 3D computed tomography data set in just a few tens of minutes. While a-Si detectors have been used with thermal neutron beams $[3,4]$, we report here one of the first implementations of such a detector for high energy $\left(E_{n}>1\right.$ $\mathrm{MeV}$ ) neutron imaging.

\section{Equipment And Facility}

The thermal neutron beam used for our imaging tests is located on Flight Path 11a in building ER-2 at the Los Alamos Neutron Science Center (LANSCE) Target-1 spallation source $[5,6]$. At LANSCE Target-1, a 10-cm diameter split tungsten target is bombarded by a $70 \mu \mathrm{A}, 800-\mathrm{MeV}$ pulsed proton beam (250 ns pulse width at 20 Hertz rate). Neutrons produced in the tungsten target are slowed down in water or liquid-hydrogen moderators after which they travel down vacuum flight tubes to the various experimental beam stations. A straight 20-m super-mirror beam guide directs the neutrons from a $\mathrm{LH}_{2}$ moderator to the experimental cave $(2 \mathrm{~m} \times 4.5 \mathrm{~m} \times 3 \mathrm{~m})$ at the end of Flight Path 11a (See Figure 1.) The beam at the end of the guide has a $6 \mathrm{~cm} \times 6 \mathrm{~cm}$ cross-section and a divergence of less than one degree. With the liquid-hydrogen moderator the energy of the neutrons ranges from less than $1 \mathrm{meV}$ to above $100 \mathrm{eV}$.

For investigations of high-energy neutron imaging we used the unmoderated LANSCE Target-4 spallation neutron source [6]. Target-4 is a water-cooled tungsten cylinder with a diameter of $3 \mathrm{~cm}$ and a length of $7.5 \mathrm{~cm}$. Neutrons are typically produced with a $5-\mu \mathrm{A}$ $800-\mathrm{MeV}$ beam delivered in $650 \mu \mathrm{s}$ long pulse trains at a rate of $100 \mathrm{~Hz}$. This source produces a broad spectrum of neutrons ranging in energy from sub-MeV to severalhundred $\mathrm{MeV}$. Neutrons emerging from Target-4 travel under vacuum for approximately $7 \mathrm{~m}$ before emerging into open-air flight paths. Our imaging tests were conducted on Flight Path 30L, which views the target at an angle of 30 degrees. The experimental station (object position) was located $40 \mathrm{~m}$ from Target-4. This distance, combined with the aspect angle of $30^{\circ}$, translates into an effective beam divergence of $\mathrm{L} / \mathrm{D}=1066$.

A number of different detectors were used with each source. For the thermal neutron source we mainly relied on a dpiX FS20 a-Si detector $(20 \times 25 \mathrm{~cm})(1536$ by 1920 pixels) having a pixel pitch of $127 \mu \mathrm{m}$ with a Bicron BC-704 ( $\mathrm{ZnS}$ with $\left.{ }^{6} \mathrm{Li}\right)$ scintillator [7]. This detector had a usable dynamic range of 2800 (>11 bits) and had only $1.5 \%$ nonfunctional

pixels. Some image-plate and film exposures were made on Flight Path 11a, but retrieval 
of the film or image plates required 15-20 minutes just to satisfy the interlock protocol and close and open the beam shutter, thus making this method of radiography unattractive for applications requiring a large number of images (such as computed tomography).

On the Target-4 flight path we used Fuji ST-VA storage-phosphor image-plates [8]. These plates were typically used in layers of eight plates interleaved with $0.5-\mathrm{mm}$ copper converter sheets. Some tests were also performed using Fuji BAS-ND image plates. These plates contain $\mathrm{Gd}_{2} \mathrm{O}_{3}$ for thermal neutron efficiency but proved to be about $1 / 10$ as sensitive to high-energy neutrons as the ST-VA plates. The image plates were scanned in a drum scanner at a resolution of $6 \mathrm{line} / \mathrm{mm}$. Scan times at this resolution were approximately 3 minutes. Image plates are very labor intensive and require manual processing after every exposure. One advantage of the image plates, however, is the large dynamic range. Exposures ranging from several minutes to several days can easily be accomodated.

For comparison to the image plates, a dpiX FS20 a-Si panel was used with a proprietary ZnS-based scintillator supplied by Industrial Quality, Inc (IQI) [9]. Several other scintillators were also tested. The relatively thick $(2 \mathrm{~mm})$ IQI scintillator was the only one tested that produced enough light to allow reasonable exposure times (60-600 s) with the flat panel in the high-energy neutron beam.

\section{Preliminary Measurements With Cold Neutrons}

Because of the lack of previous radiography development with the Target-1 source, it was unknown what deleterious effects might be produced by gamma ray contamination from the intense gamma-flash produced by proton bombardment of the tungsten target. Therefore, a number of preliminary measurements were conducted, including measurement of neutron flux, gamma flux, beam scatter, and beam uniformity. Gamma flux was measured with a Radcal Corp. Model 9010 gamma counter [10] was placed in front of the beam tube such that it covered the entire beam cross section. The flux $(3.2$ $\mu \mathrm{R} / \mathrm{s}$ ) was found to be at least an order of magnitude lower than that found in most thermal neutron beams at reactors.

In addition to measuring the gamma flux directly, a $0.0508-\mathrm{cm}$ thick cadmium foil was used to cover half of the RTM-128 a-Si detector for a $2.8 \mathrm{~s}$ exposure. The image-intensity in the region covered by the $\mathrm{Cd}$ sheet was attenuated by a factor of 2.72 compared to the half that was not covered. The area outside the beam was down by a factor of 3.74 compared to the exposed image. Later, ASTM standard blocks were used to measure the beam quality [11].

Neutron flux on the Target-1 flight path was measured with a large single-channel detector that had six tubes $(2.54 \mathrm{~cm}$ diameter, $30 \mathrm{~cm}$ long $)$ filled with ${ }^{3} \mathrm{He}$ imbedded in an $8.9-\mathrm{cm}$ thick by $23.5-\mathrm{cm}$ wide by $35.5-\mathrm{cm}$ high polyethylene block. To reduce the counting rate to the operational range of this detector, a $0.508-\mathrm{cm}$ thick cadmium sheet was placed in front of the detector to attenuate the neutron flux [12]. This detector was then gated on 10 times for $10 \mathrm{~s}$ each and the results averaged. The average number of counts per second from the $36 \mathrm{~cm}^{2}$ beam was $39,160 \pm 200$ at a proton current of about $70 \pm 2 \mathrm{~A}$. The attenuation factor of the cadmium foil was about 382 , and the detector efficiency was $18 \%$, which yields a neutron-beam flux of $2.1 \times 10^{6} \mathrm{n} / \mathrm{s} / \mathrm{cm}^{2}$. This 
compares favorably with the neutron flux $\left(6 \times 10^{5} \mathrm{n} / \mathrm{s} / \mathrm{cm}^{2}, \mathrm{~L} / \mathrm{D}=190\right)$ at the decommissioned (1993) Omega West reactor, where neutron radiography was previously performed at Los Alamos [13].

Given the beam apertures and distances back to the moderator, the $\mathrm{L} / \mathrm{D}$ ratio was calculated to be approximately 43 . An exact $\mathrm{L} / \mathrm{D}$ is not a trivial calculation due to low angle reflections of neutrons in the beam guide and the uncertainty in the neutron spectrum. A beam divergence of less than one percent is expected for subthermal neutrons.

A measure of the scatter at the detector and at other areas was made by counting the number of hits found in the detector pixels that were not exposed directly to the beam and by moving the ${ }^{3} \mathrm{He}$ detector to other locations in the cave. The scatter was equal to about $5 \%$ of beam flux used to image our samples and could be reduced by replacing the beam stop in the experimental cave.

To determine beam uniformity, the RTM-128 a-Si detector, and R-type x-ray film (with a Gd converter foil) were exposed at the exit of the beam guide to produce images that were evaluated for uniformity. The beam at this location appears to be uniform to $\pm 5 \%$ [see Figure 2 (left)]. Data from the 42nd row and column are shown as solid and dashed lines in Figure 2 (right). (The 42nd row and column were chosen because they bisected the beam cross-section.) The line plots show a fairly even distribution across both axes of the detector.

\section{Imaging Tests With Cold and Thermal Neutrons}

Due to limited beam availability we were only able to obtain a few images with the a-Si detectors. The objects imaged were a 3/8-inch stainless-steel bolt threaded through a brass nut and a 1/4-inch thick stainless-steel test piece. The test piece had several holes and slots machined half way through the thickness of it. Centered in each of the holes and slots, smaller holes and slots were machined completely through the thickness of the piece.

The exposure lengths for the images varied between 2.8 and 5.6 seconds. In some cases, as many as 50 exposures were taken and the data were averaged to get better statistics. However, the quality of the multiple exposure images was not much better than a single frame exposure.

On the left in Figure 3 is an image taken with a Bicron BC- $704{ }^{6} \mathrm{Li}$ doped $\mathrm{ZnS}$ scintillator to be compared with the image on the right taken with a Rarex scintillator.

The images taken with the BC-704 scintillator were significantly sharper and had more contrast than the Rarex scintillator. However, the Bicron scintillator did show radiation damage after 12 hours of exposure to the beam. The image of the test piece in Figure 3 shows the test piece held in place next to a stainless steel bolt. The image shows several holes and the slot milled partially through its thickness. However, it doesn't resolve the smaller holes that are milled completely through it. This is at least partially due to the fact that the holes and slots (which go completely through the thickness of the material) were smaller than the pixels of the RTM-128 a-Si panel. 
To produce typical CT images (such as the one shown in Figure 4.) a rotation stage was stepped in 0.5-degree increments and images recorded with the a-Si panel. Image acquisition and transfer time was about 7 seconds per image, however it took about an hour or more to obtain a data set in this manner because the rotation and image acquisition had to be initiated manually. Due to software problems, the data sets were not optimized for dynamic range and therefore do not indicate the best image that can be achieved with this beam line. Because of the sparse data set, a number of artifacts are visible, but the rind artifact due to beam hardening is not apparent.

\section{Imaging Tests With High-Energy Neutrons}

The neutron beam diameter at the 40-m experimental station on Target-4 Flight Path 30L is approximately $20 \mathrm{~cm}$. Storage-phosphor image plates have been used at this location to investigate applications of high energy neutron radiography. Detector efficiencies with thin converter layers are considerably smaller for high-energy neutrons $\left(E_{n}>1 \mathrm{MeV}\right)$ than for thermal neutrons, leading to longer exposure times to obtain equivalent statistical quality. Image plates are well-suited for testing under these conditions because they can be set in place and left unattended for many hours without active monitoring. Various beam and detector properties were investigated, including spatial resolution, high-energy gamma-ray contamination, background from forward-scattered and inelastic neutron scattering, beam stability, image-plate response stability, and contrast-limiting factors intrinsic to the image plates and scanning process.

The Target-4 neutron flux was not measured directly, but rather was calculated from Monte Carlo simulations that have been benchmarked against fission-chamber measurements in the lowest energy $\left(E_{n}<100 \mathrm{MeV}\right)$ region [6]. For normal beam conditions $(5 \mu \mathrm{A}$ current), with a $6-\mathrm{mm}$ lead gamma filter, and $33 \mathrm{~m}$ air path, the flux integrated over the energy range from $1-800 \mathrm{MeV}$ is approximately $1 \times 10^{6} \mathrm{n} / \mathrm{s} / \mathrm{cm}^{2}$. This is very close to the flux obtained on the Target-1 Flight Path 11a with thermal neutrons. The main difference in count rates is therefore due to the much lower detector efficiencies for high-energy neutrons.

Some modest successes were achieved with the image plates, including successful detection of holes in $0.5-\mathrm{mm}$ and $0.8-\mathrm{mm}$ plastic sheets shielded by a variety of higher-Z materials, including up to $5 \mathrm{~cm}$ of depleted uranium. A tomographic image of a flat-plate test object was also obtained, and showed good agreement to a model image generated by ray-tracing techniques employing total neutron cross sections. A comparison between the model and image-plate data is made in Fig. 5.

Further modeling of this flat-plate test object was done with the recently released MCNP4C code with the radiography patch (X patch) [14]. Our version of the MCNP code has the ability to rotate the object and obtain an arbitrary number of two dimensional views that can be assembled into sinograms for further processing by our CT codes. In this case, monoenergetic $10-\mathrm{MeV}$ neutrons from a plane-beam incident source were transmitted through the part with 400 by 400 pixel resolution $(0.75 \mathrm{~mm}$ per pixel) for 200 views in 360 degrees. The scattering was turned off in MCNP, so that this is equivalent to a ray tracing with the correct total cross sections as in Figure 5 but with more views and volume imaging. The total running time in MCNP on eight processors 
on Theta (a SGI cluster on Blue Mountain) was 22 minutes for the 200 views. Full volume reconstruction (Figure 6) took 5 minutes on a cluster of ten $350 \mathrm{MHz}$ Pentium 3 processors.

Resolution in the high-energy images was limited by the finite size of the Target- 4 source and by the reaction cone of neutron scattering in the converter and phosphor layers. We measured the line spread function of the system by differentiating the edge profile obtained from a $5-\mathrm{cm}$ thick uranium cube carefully aligned parallel to the beam. The result is best described by a Lorentzian line shape with a full width at half maximum of $0.75 \mathrm{~mm}$. This result was obtained with the image-plate stack (8 layers) separated from the test object by a distance of $50 \mathrm{~cm}$. This large stand-off distance is required to minimize contributions from forward-peaked beam scattering.

Because of the manual processing required, image plates are not suitable for high-volume imaging applications such as tomography. In addition, our tests showed that some contrast-limiting features such as surface texture in the protective front layer make image plates unsuitable for applications looking for low-contrast features with spatial dimensions close to the overall system resolution.

As a potential replacement for the storage-phosphor image plates, we tested a dpiX FS20 flat-panel array with several different scintillators. The best results were obtained with a 2-mm thick ZnS based scintillator obtained from IQI. Comparison images of a steel and brass padlock and BNC terminator obtained with the a-Si panel and a four-layer stack of image plates is shown in Figure 7. The exposure time with the a-Si panel was about 7 minutes and the exposure time with the image plates was about $11 \mathrm{~min}$. Both images are corrected for field curvature by normalizing to object-out exposures.

\section{Conclusion}

With a limited amount of beam time we were able to characterize several beam properties as well as produce images of test samples in both thermal and high-energy neutron beams. The resolution of the images in the thermal neutron beam was limited only by the pixel size of the a-Si detector. Resolution in the high-energy neutron beam was limited by source-size, standoff distance, and scintillator thickness and was much larger than the a-Si pixel size.

Imaging with the a-Si detectors has been shown to produce quality digital images in a short amount of time with minimal setup, making it an attractive detector for many applications. No degradation in the detector in the thermal neutron beam was noticed during the collection of these images, however the scintillator material did suffer some discoloration and subsequent efficiency degradation. Exposure of the a-Si panel to highenergy neutrons was limited to a total of about $45 \min$ (approximately $3 \times 10^{9} \mathrm{n} / \mathrm{cm}^{2}$ ), so the effects of radiation damage in this type of beam have not yet been fully tested.

\section{Acknowledgements}

We would like to thank Steve Sterbenz, Kari Smith, Ken Griechen, Ed Snow ,Gene Farnum, and Art Bridge who assisted in one way or another with this project. This work was supported by the ESP (Enhanced Surveillance Program) at Los Alamos. 


\section{References}

1. EG\&G Amorphous Silicon, 2175 Mission College Blvd., Santa Clara CA, USA

2. dpiX, LLC, 3406 Hillview Ave., Palo Alto CA., 94304, USA; www.dpix.com.

3. G.W. Fraser, “Thermal Neutron Imaging”, Proc. SPIE Vol.2339, 287-299, (1994).

4. T. N. Claytor, M. J. Schwab, E. H. Farnum, T. E. McDonald, W. L. Sievers, M. J. Sheats and D. M. Stupin, "Thermal and Cold Neutron Imaging at the Los Alamos Neutron Scattering Center Using an a-Si Detector Array", Los Alamos Report LAUR 97-733 (1997).

5. G. J. Russell, R. P. Hjelm Jr., T. O. Brun, E. J. Pitcher, and L. L. Daemen, "Los Alamos Pulsed Spallation Neutron Source Target Systems", Trans. of the Am. Nuclear Soc. V69 pp 431-432, (1993).

6. P.W. Lisowski, C.D. Bowman, G.J. Russell, and S.A. Wender, "The Los Alamos National Laboratory Spallation Neutron Sources", Nucl. Sci. and Eng. 106, 208 (1990)

7. Bicron Newbury, 12345 Kinsman Road, Newbury, OH 44065-9577, USA; www.bicron.com

8. Fuji Photo Film Co., LTD, 26-30, Nishiazabu 2-Chome, Minato-ku, Tokyo 106, Japan; http://home.fujifilm.com/products/science/index.html

9. Industrial Quality, Inc., 640 E. Diamond Ave., Suite C, Gaithersburg, MD 208775323; USA; www.indqual.com

10. Radcal, Corporation, 426 West Duarte Road, Monrovia, CA 91016, USA; www.radcal.com

11. "Standard Method for Determining Image Quality in Direct Thermal Neutron Radiographic Examination",Vol.03.03 Nondestructive Testing, ASTM E 545-91, (1994).

12. H. O. Menlove, M.M. Fowler, E. Garcia, M.C. Miller, M. A. Paciotti, R.R. Ryan, S.E. Jones, “A High-Performance Neutron Time-Correlation Counter", Nucl. Tech.. 71, 497-505, (1985)

13. T. H. Feiertag, "Thermal Neutron Radiography at Los Alamos", Los Alamos Conference Proceedings, LA-11393-C (1988).

14. Judith F. Briesmeister, Editor, "MCNP- A General Monte Carlo N-Particle Transport Code," Los Alamos National Laboratory report LA-13709-M (April, 2000); http://laws.lanl.gov/XCI/PROJECTS/MCNP/manual.html; L.S. Waters, MCNPX User's Manual, Version 2.1.5, TPO-E83-G-UG-X-00001, November 14, 1999. 


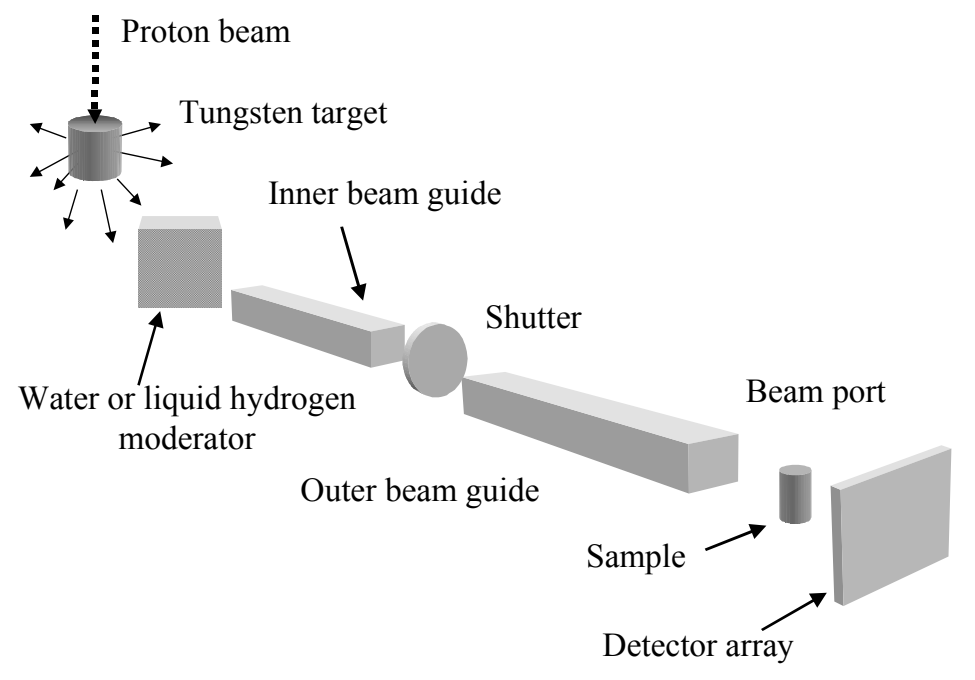

Figure 1. Schematic diagram of the experimental setup with cold neutrons at LANSCE target 1. 

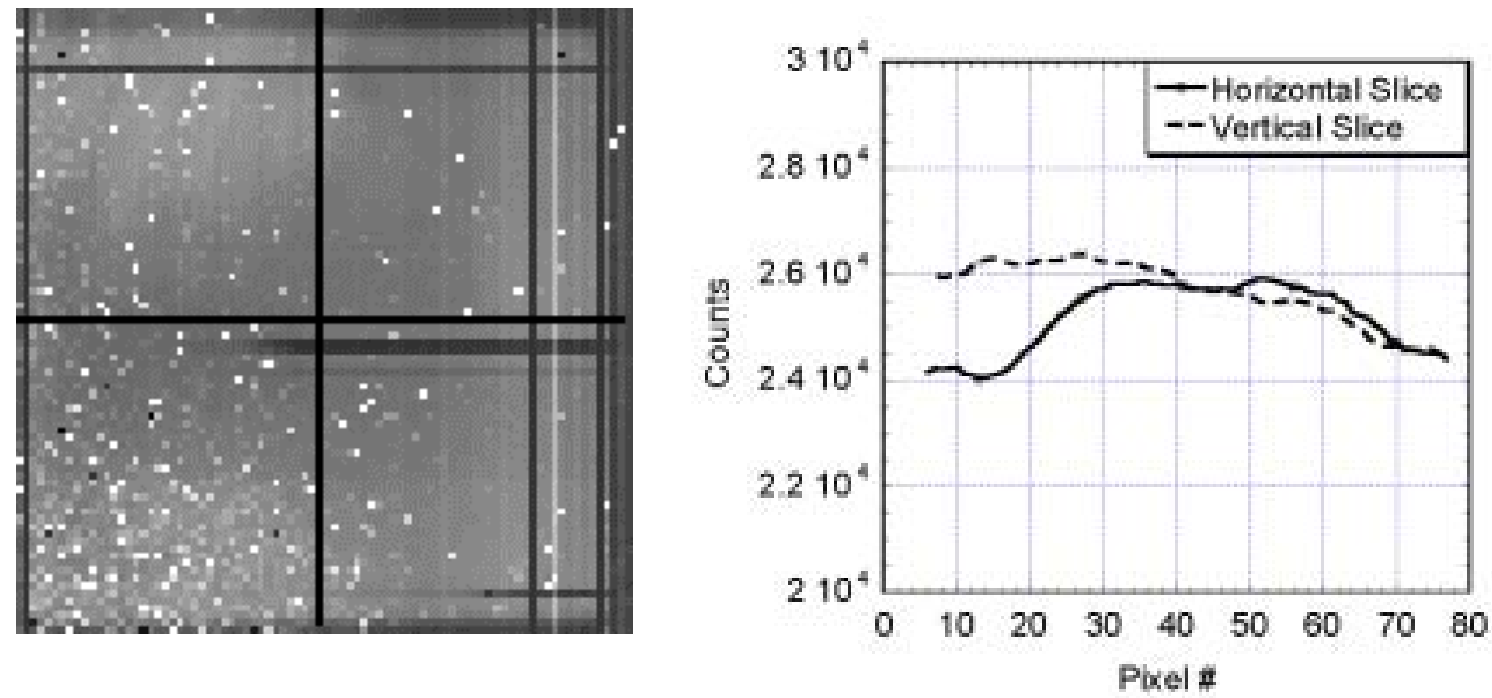

Figure 2. The image on the left is of the LANSCE Target-1 FP11a beam at the beam guide exit (as measured with the RTM128 and BC 704 scintillator) with no test object. The two black intersecting lines represent the row and column presented in the smoothed line plot in the right hand image. Other spots and lines, in the image on the left, are defective or weak pixels or lines. 

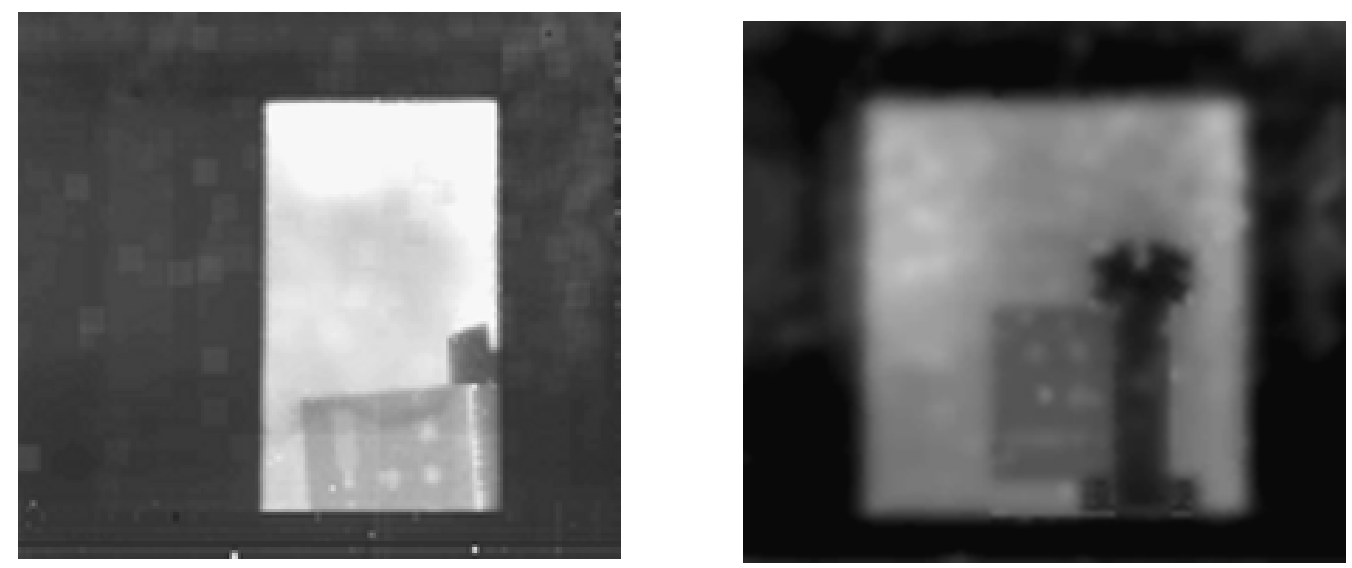

Figure 3. On the left is an image of the beam using the RTM-128 a-Si detector and BC704 scintillator with the left-hand-side of the detector covered with a $0.05 \mathrm{~cm}$ thick $\mathrm{Cd}$ foil. Notice that the part of the image that is covered is almost as dark as the area around the beam where there is little to no neutron flux. On the right is an image of the same bolt and test piece using a Rarex (gadolinium oxysulfide doped with terbium) scintillator. 

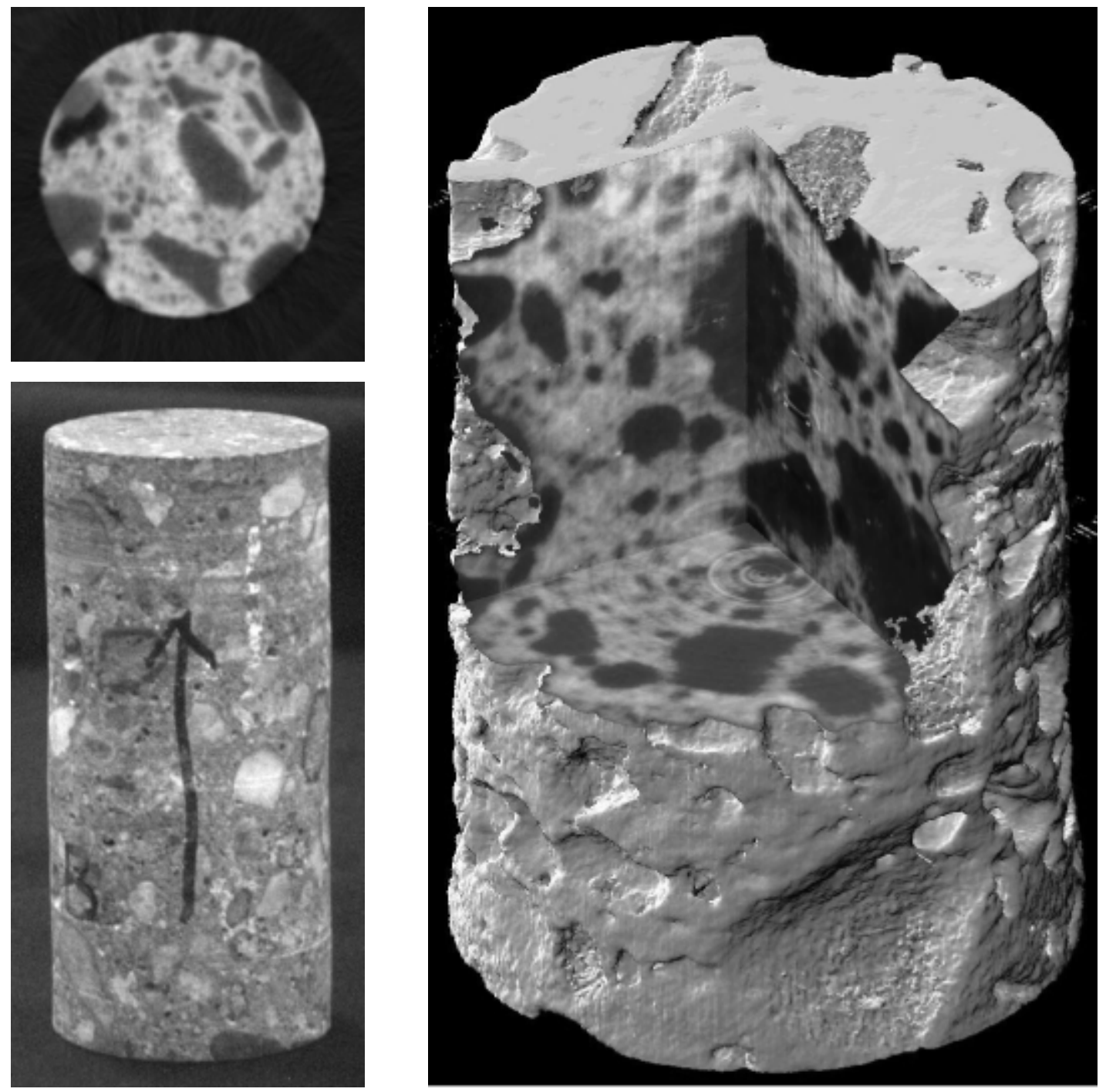

Figure 4. Concrete core sample (lower left) and cut-away view of a 3D tomographic reconstruction (right) based on 360 views with an dpiX FS-20 a-Si detector. On the upper left is a $2 \mathrm{D}$ cross section. 

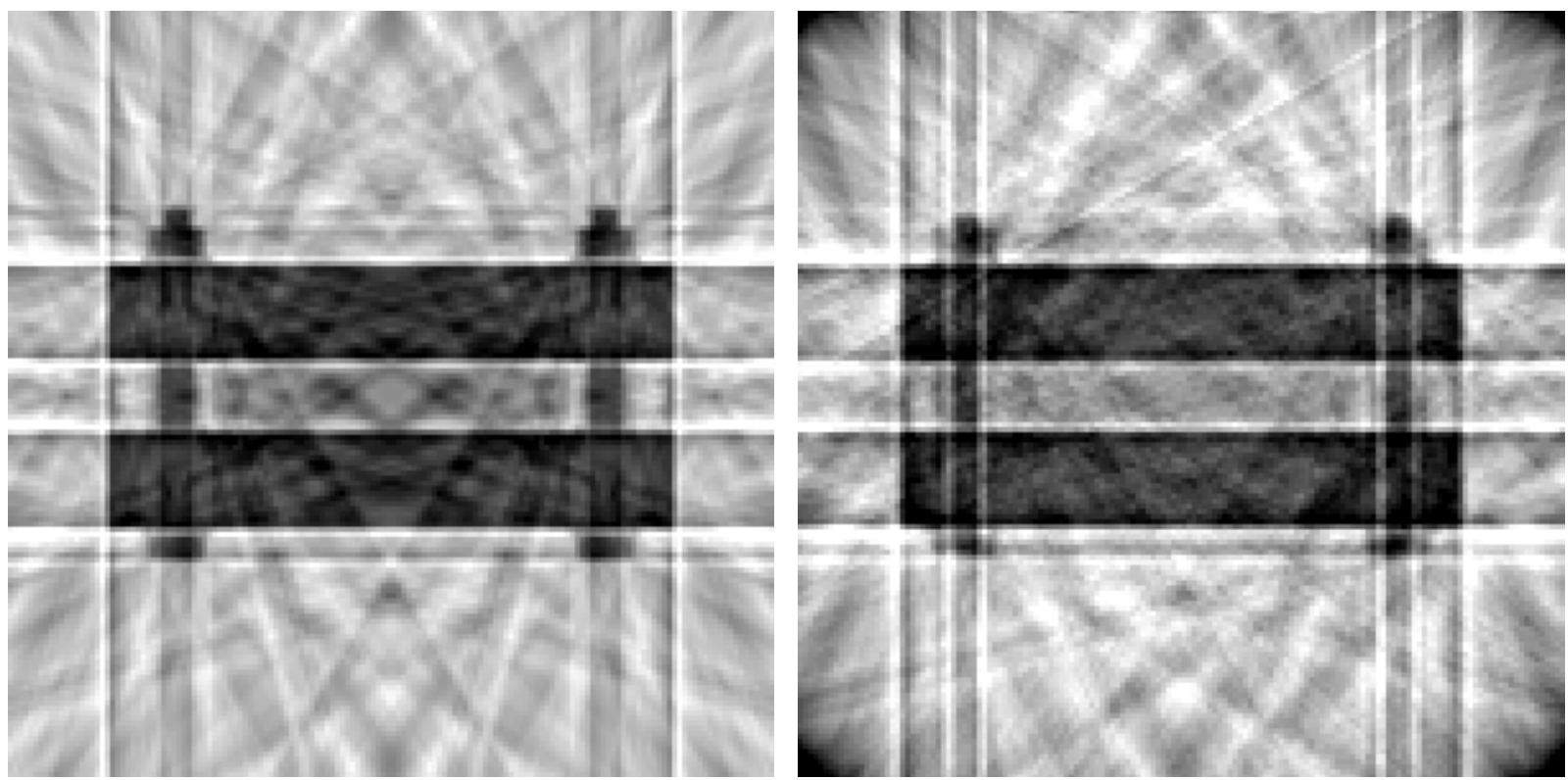

Figure 5 Tomographic reconstruction of an assembly of two3/4-inch iron plates held together with 1/4-20 bolts. The left image is a ray-trace simulation and the right image is a measurement made using storage phosphor image plates and high energy $\left(E_{n}=1-800 \mathrm{MeV}\right)$ neutrons. The reconstructions are based on 12 views ( 15 deg steps) in a plane through the bolts. 


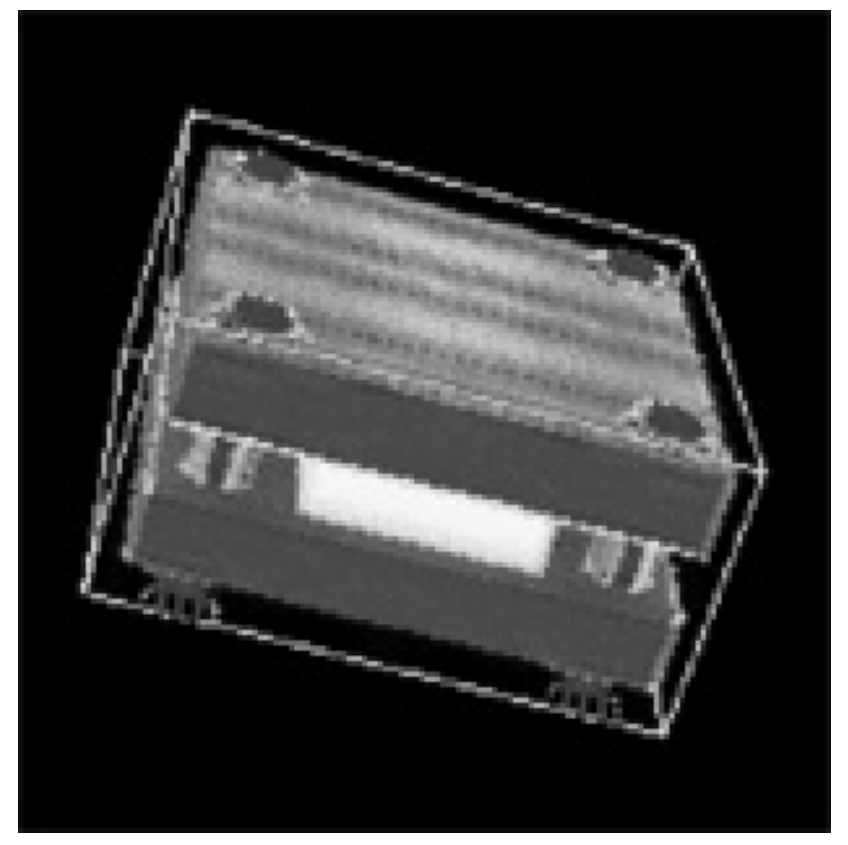

Figure 6. 3D tomographic reconstruction of a flat-plate test object based on 200 views generated with the Monte Carlo code MCNP4C. The resolution of the 400 $\times 400$ pixel $2 \mathrm{D}$ views was $0.75 \mathrm{~mm}^{2} /$ pixel. 

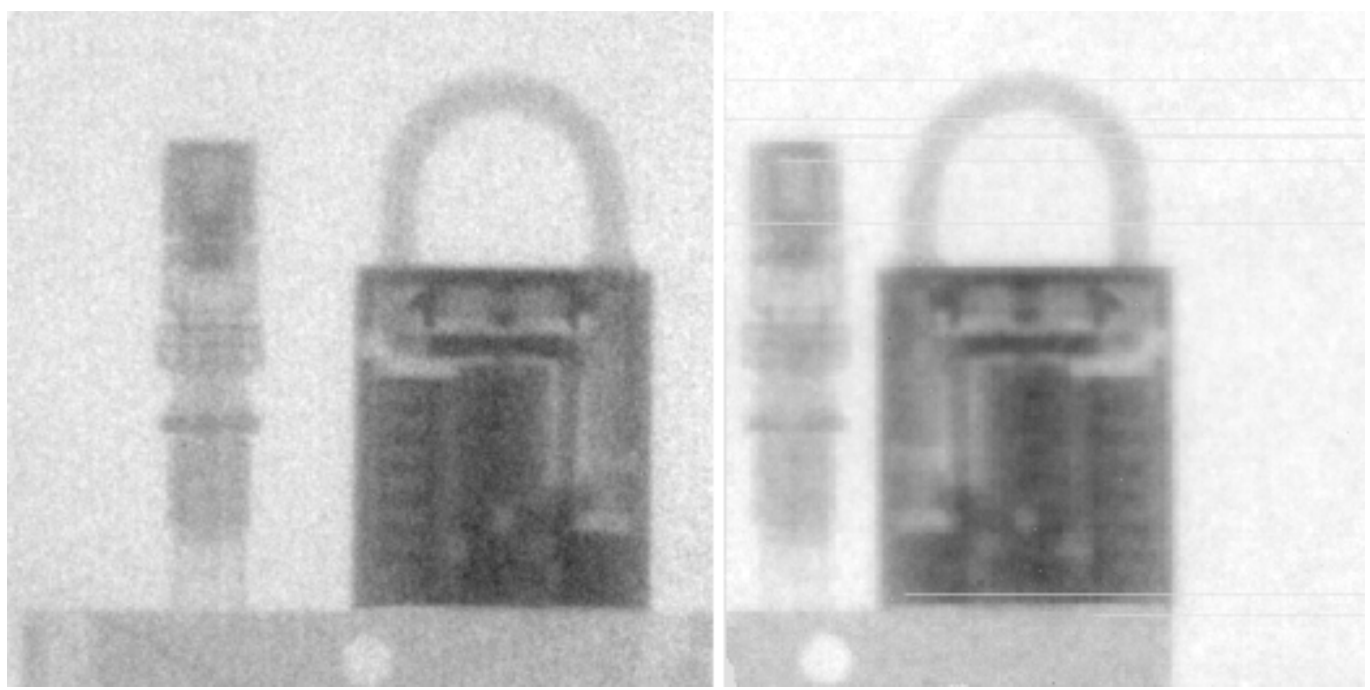

Figure 7. Comparison images of a BNC terminator and padlock obtained with high-energy neutrons $\left(E_{n}=1-800 \mathrm{MeV}\right)$ and storage-phosphor image plates (left) and an amorphous-silicon panel detector (right). 